\title{
Reflection and Comparison of the Eastern and Western Aesthetics of Photography Composition
}

\author{
Bochen Zhang * \\ Shandong University of Arts, Jinan 250014, China \\ bochenart@126.com
}

Keywords: Sino-western culture; aesthetics; painting.

\begin{abstract}
The purpose of this paper is to study the different skills of photography composition by comparing the sino-western aesthetics. On the basis of sino-western culture, sino- western classical painting is taken as the comparative object to study the differences between all kinds of aesthetics. The method is to design a question consciousness as the research the aesthetic difference. Therefore, the five aspects esthetics, such as the sino-western classical painting esthetic characteristic, the spatial consciousness, the drawing subject matter, the color idea and the development road are compared and researched. After the research, different cultural connotations and aesthetic characters of sino-western classical paintings are revealed, and an attempt is made to explore the developing direction of contemporary sino-western painting. The contrast result shows that there are great differences between sino-western aesthetics thought. Based on the above finding, it is concluded that it is the best interpretation to respect the culture difference and learn sino-western aesthetics modestly.
\end{abstract}

\section{Introduction}

Painting is a highly personalized artistic expression and a manifestation of human civilization. When Mr. Chen talks about civilization in the book Civilization \& Culture, he believes that the significance of civilization lies in the fact that man uses science, technology and other means to transform the objective world. People harmonize group relations to regulate their emotions through law, morality and other systems, and use religion, art and other forms. This behavior meets the basic needs to the greatest extent and achieves the level of overall development. From the cultural point of view, the sino-western classical paintings have profound implications for the value and significance of painting [1]. From this perspective, the research on painting is not only the study method of painting in a concrete way from the experimental point of view. It means that all the artistic forms in painting are regarded as symbols of culture. Culture is a relationship, convention and system that expresses the intrinsic attributes and characteristics of anything. Through culture, people can better look at the characteristics of the times, the regional style and the national style of the people in the process of transforming the objective world, coordinating the group relations and regulating their emotions [2]. Chinese classical painting refers to the paintings during the period from Wei Jin to the late Qing dynasty. The works of this period mainly take scroll painting as the research focus, but also involve paintings, murals and other painting forms. Western classical painting mainly refers to the works that started in ancient Greece and ended in Impressionism in the late nineteenth century. It mainly focuses on murals and oil paintings, but it also deals with paintings such as wall mosaics. But it is necessary to point out that culture is not the only reason for the difference between sino-western painting concepts. The differences between sino-western painting concepts should be the result of the resultant of many factors. Culture, as a kind of soft power, should have important value and function [3].

The sino-western classical painting is analyzed from the cultural point of view. Taking sinowestern painting as the materialization of human civilization, the differences between sino-western painting language are explored [4]. Then the in-depth research for the cultural and aesthetic study is carried out and the reasons for the diversity of the language forms are explained. This study tries to find out the shaping function of sino-western culture from different levels and angles, and makes comments on the direction of the development of contemporary painting. 


\section{The aesthetic pursuit of sino-western photography composition}

Photographic art is an important component of culture, and it is the bearer of human civilization, with distinctive features of the times and national characteristics. In ancient times, the aesthetic pursuit of sino-western art was quite different. The aesthetic pursuit of Chinese classical art presents the trend of dynamic development, such as the development from vivid portraiture to rhythmic vitality [5]. The aesthetic pursuit of Chinese classical art with heavy verve continues to develop and expand. Taking the relationship of form and spirit as the core, the aesthetic category in classical aesthetics history China gradually bred and mature [6]. The aesthetic pursuit of Western classical art runs through the ancient art history of ancient Greece, Rome, middle ages, Renaissance and seventeenth century. Western classical art pays attention to the beauty of form and pays attention to the imitation of the outside world. It sets up a model of ideal beauty, and hopes to express eternal beauty through painting. The western classical painting eventually formed a realistic painting system. The differences between sino-western art pursuits come from the differences between sino-western classical aesthetics. Both the classical aesthetics are harmonious aesthetics, but their connotations are different and need to be screened out.

\subsection{The aesthetic pursuit of China}

Chinese classical painting is the carrier of the spirit of Chinese classical aesthetics. It has the unique value and status in the history of Chinese aesthetics, which is the most artistic way to show the spirit of Chinese classical aesthetics [7]. The aesthetic pursuit of Chinese classical painting is profound and pluralistic, and different aesthetic categories are put forward in different historical periods. The aesthetic concept of Chinese classical painting is constantly developing and expanding. It has a profound cultural implication and connotation. Naturally, China's photographic composition skills also follow the traditional style of painting. One of the most exquisite is the contrast and balance of photography. In the composition of photography, the basic law of form beauty is varied and unified, which is embodied in two aspects, that is, contrast and balance. In a sense, the art of photographic composition is a comparative art. Contrast is the necessary condition for the diversity and variety of pictures [8]. At the same time, it is an important means of highlighting the theme and one of the major rules of form beauty. Proper use of contrast can make the theme more vivid and enhance the artistic appeal of the picture.

Contrast and balance are two basic methods commonly used in photography to achieve formal beauty. These are two different methods of expression, but they are interrelated and interdependent. For example, if the photographer only uses the contrast to highlight the main image and does not balance the primary and secondary, then the picture is disorganized and the beauty is lacked. However, if they only consider balance without contrast, primary and secondary, then the picture will appear dull and inflexible. Generally speaking, the picture of beauty needs balance in contrast and contrast in balance. For all photographic compositions, contrast and balance need to be taken into consideration. Although some pictures are more balanced, the contrast is not obvious or more prominent, and the balance is not significant. The unity of contrast and balance can form a picture of diverse and unified forms.

\subsection{The aesthetic pursuit of western countries}

Western classical painting refers to the whole western painting art from the ancient Greek to the late nineteenth Century. The painting of this period underwent the changes of Greek, Gothic, Byzantine, Baroque, Rococo, Neo classicism and romantic styles. But in the general aesthetic pursuit, it has consistent basic characteristics. That is, it pays attention to the beauty of form and the imitation of the outside world, forming a model of beauty. It wants to express eternal beauty by painting. The beauty of idea is at the core in the aesthetic concept of classical painting. Therefore, the western photography composition also pays attention to the discovery and expression of the esthetic sense color.

As early as in the eighteenth century, western photographers laid down the foundation for the emergence of photographic technology for the study of camera obscura and photographic materials. One of the earliest existing pictures was a blurred image of a sensitive asphalt board taken by a photographer in 1827 from a window and images of buildings in the sun. For the purpose of shooting, 
this photo is intended to objectively record streets and buildings, so it can be considered as the first architectural photography.

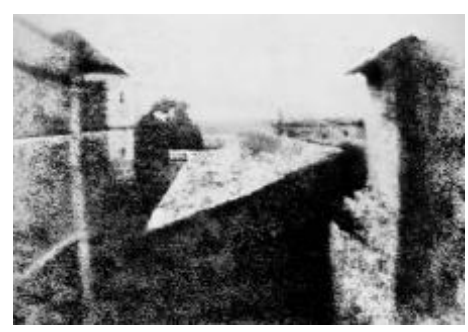

Figure 1 View from the Window at Le Gras

\subsection{Different of the harmonious beauty of sino-western photography composition}

The harmonious beauty of Chinese and Western classical painting is a feeling of painting itself, which includes two layers inside. The external structure of painting is the symmetry, proportion and order of objects, or rhythm and harmony. The internal structure of painting is the cultural spirit embodied in the form. Western classical painting attaches great importance to the objective form which can be perceived by the senses. Emphasis on the aesthetic object is the harmony of physics, while Chinese classical painting pays attention to the choice and promotion of the painter's personality to the external nature. It is an expression of the artist's personality, mainly embodied in the inner charm of painting. Paying attention to the psychological harmony of the aesthetic subject reflects the different pursuit of harmony between sino-western classical painting.

Western classical painting pays attention to form and pursues external truth which can be sensed by sensory organs. Just as some critics have said, "beauty is a form that can be perceived by the senses." This is the important reason why Western art, especially the western traditional painting, emphasizes on satisfying the beauty of visual reality. However, the extroverted characteristics of thinking make the art change constantly in the ways and means of imitation and imitation." This realistic form of painting is conceived by the harmony aesthetics and imitation theory of ancient Greece, and is the product of the harmonious aesthetics of ancient Greece.

\section{The spatial view of sino-western photography composition}

In the long-term historical and cultural development, the consciousness of time and space in China and the west is very different. Therefore, the space concept of sino-western painting is also different. The Chinese are the space-time view of life, and they convey charm in painting. The westerner is the physical spatial view, and they display the modelling beauty in the painting. Therefore, the concept of space in photography is a comprehensive reflection of philosophical thinking, mode of thinking and aesthetic psychology. Based on the different cultural backgrounds of China and the west, there are obvious differences in the spatial connotation, composition and aesthetic charm of sino-western photography.

\subsection{The beauty of space in Chinese photography composition}

The function of lines

The spatial sense of Chinese photography embodies the function of online modeling at first. It is different from the western architecture in the form of shading and shading. Chinese photography inherits the construction techniques of classical painting. It never uses light and shade to construct pictures, mainly using lines to form pictures. Chinese art takes the line as the modelling method of painting, and is influenced by Chinese calligraphy creation. For example, Sheikh put the pen and bone together in painting. This communicated painting and calligraphy, and achieved the beauty of the lines of classical painting.

Lines are used to shape pictures, and Chinese classical paintings have the tendency to blur the depth and space. Different from the reconstruction of the three-dimensional depth space in the west, the Chinese classical painting develops to the plane in space. Chinese traditional painting shows the space by means of images. Both the performance of China traditional painting space form fuzzy depth space construction. The dialectics of opposites just conforms to the performance of the two- 
dimensional space, and becomes the internal law of expressing the space by stylized expression, and achieves the goal of artistic expression of unlimited rich practical space.

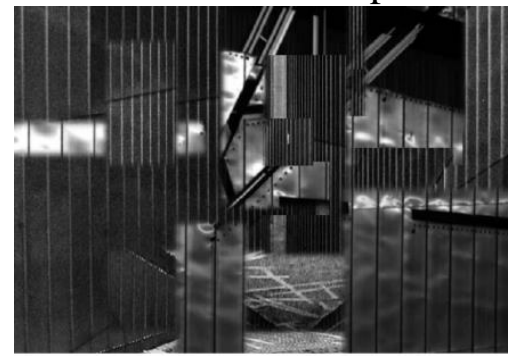

Figure 2 The background structure of lines

Photography aesthetic space

The painting space shows the characteristics of the three-dimensional space in the two-dimensional plane. The western classical painting has achieved the reconstruction of the deep space through the use of perspective, shadow, light and shade and color. Chinese classical paintings ignore the focus of perspective, while using line modeling. The walking sight is used to create habitat composition and focus on the line. China art usually adopts the means of opening and closing, momentum to solve space problems, thus forming the unique aesthetic space of Chinese classical painting. 而Painting was originally a kind of art of space. When the time factor is introduced into space, the space of painting is endowed with a new meaning. Painting was originally a kind of art of space. When the time factor is introduced into space, the space of painting is endowed with a new meaning. The aesthetic experience has changed in fundamental. Chinese classical painting unfolds in the form of "vertical axis" or "long scroll", and the space presents dynamic changes in the rhythm of time, forming the dynamic beauty of Chinese classical painting.

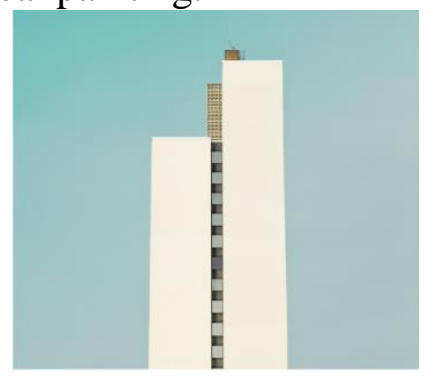

Figure 3 The space expression

\subsection{The beauty of modeling in western classical painting}

Painting is a kind of space art, so the painting space is different from the reality space. In painting, the spaces that organize harmony are not through vision or touch, free movement or suppression, far or near the sound, the disappearance or the echo of the conscious space. The space that can be perceived by motion or sound by sight or touch is the real space. The actual space is accessible, measurable, and specific. The space of painting is virtual space. The space of painting is only a visible object, and it does not exist for the tactile, auditory and muscular activities. For them, painting is just a fairly small flat canvas, or a cold, nothing wall. For the eyes, it is a bottomless space full of shapes. This pure visual space is an illusion. The space of painting is a visual illusion. Although people cannot enter or measure it, it can give person a kind of illusion in the virtual. In particular, western paintings use the method of focus perspective, trying to create an illusion of three-dimensional space, so that the western classical painting formed a three-dimensional space modeling beauty. Although painting space is different from the reality space, it is closely related. Painting space is the creation of real space.

Although painting space is virtual space, it has its own independent life, and is self-contained. Illusory space, as a completely independent thing, not a part of the actual space, is an independent and complete system. Whether it is two-dimensional or three-dimensional, it can continue in all its possible directions, with infinite plasticity. Although the space of western painting is a kind of physical space, it also has vitality. Life is the soul of all art. But compared with Chinese classical painting, western classical painting is more satisfied with a vivid reproduction of a physical space. 
Therefore, the beauty of western classical painting refers to the three-dimensional space. It can measure and enter the spatial illusion and the emotion of the artists who fill it. Formation and emotion together form the beauty of Western classical painting.

\subsection{The contrast principle in photography composition}

The principle of contrast is to use the difference between the elements of the same element in the picture to serve the subject. The elements are divided into four kinds: graphic contrast, contrast of light and shade, color contrast and virtual-real comparison.

First, graphic contrasts are the use of dots, lines, and elements of a painting or photographic work to represent the subject in terms of quantity, location, size, and character. For example, a building can be easily represented in a context consisting of a form element, an online element, or a point element.

Second, light and shade contrast is the most common form of contrast in architectural photography. As a product of light, it is inevitable in architectural photography, while architectural photographers can control the contrast and serve as a theme

Third, color contrast is reflected in the four attributes of color. That is, brightness contrast, saturation contrast, hue contrast and tonal contrast. The contrast of these color attributes can be presented either alone or simultaneously in the picture to satisfy the requirements of the theme expression.

\section{Conclusion}

By comparing the differences between eastern and Western cultures and photographic composition, the following conclusions are drawn. First, the photographic picture should be able to condense the attention of the viewer, so as to form a center of interest that attracts great attraction. Second, the picture should be clear and fresh. The traditional picture form is hard to bring up people's interests. However, novel and unusual objects excite the nervous system and stimulate intense attention. Third, the creative expression of photography composition is to break through the existing forms and to dare to be different. The picture form of a photographic work can never be a permanent pattern even if it is better. From the point of view of artistic dialectics, the paradigm is always relative, and the breakthrough is absolute. Art forms are also evolving. Finally, it is concluded that different social culture will lead to different aesthetic pursuit, so seeking common ground while reserving differences and common development are the best expressions of cultural identity.

\section{References}

[1] Piotrowska, A. G. (2016). About twin song festivals in eastern and western europe: intervision and eurovision. International Review of the Aesthetics \& Sociology of Music, 47(1), 123-135.

[2] Wang, W. N., Liu, J. C., Xu, X. M., Jiang, Y. Z., \& Wang, L. (2015). Aesthetic enhancement of images based on photography composition guidelines. Huanan Ligong Daxue Xuebao/journal of South China University of Technology, 43(5), 51-58.

[3] Zhang, Y., Huang, Q., Qin, L., \& Zhao, S. (2015). Strategy for aesthetic photography recommendation via collaborative composition model. Iet Computer Vision, 9(5), 691-698.

[4] Järdemar, \& Cecilia. (2016). Photography and the face: the quest to capture the contained. International Journal of Language \& Communication Disorders, 40(2), 137-49.

[5] Paldino, S., Bojic, I., Sobolevsky, S., Ratti, C., \& González, M. C. (2015). Urban magnetism through the lens of geo-tagged photography. EPJ Data Science, 4(1), 5.

[6] Yang, Y. H. (2015). Territories and world: the migration and variation of chinese literati aesthetics in modem east asia. , 42(11), 181-200.

[7] Lopes, M. I. (2015). Aesthetics of interaction in digital art. The British Journal of Aesthetics, $55(2), 261$.

[8] Little, D. (2015). The impact of aesthetics in restorative treatment planning. Dentistry Today, 34(5), 245-259. 